\title{
Review: Adaptation in placental nutrient supply to meet fetal growth demand: Implications for programming
}

\author{
Colin P. Sibley*, Paul Brownbill, Mark Dilworth, Jocelyn D. Glazier \\ Maternal and Fetal Health Research Group, School of Clinical and Laboratory Sciences University of Manchester, Manchester Academic Health Science Centre,
} St Mary's Hospital, Manchester, M13 9WL, UK

\section{A R T I C L E I N F O}

Article history:

Accepted 17 December 2009

\section{Keywords:}

Nutrient

Transport

Programming

\begin{abstract}
A B S T R A C $T$
This review considers the hypothesis that adaptations in blood flow, exchange surface area and transporter activity enable placental supply capacity to meet fetal demand and cause alterations in fetal composition which result in life-long programming of homeostatic set points. We consider the components of placental supply capacity and describe the predominant changes each of these could impose on solute and water exchange across the placenta. We next consider the evidence that adaptations in placental nutrient supply to meet the demands of fetal growth and development do occur. Evidence from human and mouse studies suggests that adaptations occur in regulation of blood flow through the fetoplacental circulation, in exchange barrier surface area and in transporter-mediated processes for amino acids and calcium. Crucially there appear to be differences in the gestational timing of these adaptations. Finally we suggest that each of these adaptations could have separate effects on the composition of the fetus. These could affect physiological set points in different ways and so programme the lifetime responses of the individual.
\end{abstract}

(c) 2010 Published by IFPA and Elsevier Ltd.

\section{Introduction}

The main purpose of this review is to advance the hypothesis that the capacity of the placenta to supply nutrients can adapt, or is modified, to meet the nutrient requirements of the fetus. In critical periods of some pregnancies the flux of nutrients across the placenta (e.g. in mol solute/g placenta/min) is insufficient to meet the requirements of the fetus for growth and development. This limits the ability of the fetus to achieve its growth potential. The opposite can also occur - the flux of nutrients across the placenta may exceed that actually needed by the fetus. If unchecked the former situation leads to a failure of the fetus to achieve its genetic growth potential, which if severe leads to the clinical situation of fetal growth restriction (FGR) [1]. The latter situation leads to fetal overgrowth or macrosomia, such as is often found when pregnant women are diabetic or obese [2]. Both fetal undergrowth and overgrowth can lead to disease sequelae, both in the neonatal period and later in life, as has been well documented elsewhere [3]. Adaptation in placental supply capacity to more exactly match fetal demand could be a homeostatic mechanism preventing fetal undergrowth or overgrowth occurring more often than it actually

\footnotetext{
* Corresponding author. Tel.: +44 0161276 6484; fax: +44 01612241013.

E-mail address: colin.sibley@manchester.ac.uk (C.P. Sibley).
}

does. However, as is reviewed below, there are many different mechanisms by which placental supply capacity may be modified and which do indeed appear to occur. The combined effect of each of these modifications, in terms of increasing or decreasing total nutrient net flux across the placenta, might be the same but the proportional effects on the transfer of specific nutrients could be quite different. This could result in altering the relative proportions of the different constituents (fat, muscle, metabolite concentrations in fluid compartments) of the delivered baby from what they otherwise might have been - with effects on the programming of homeostatic set points.

This review will consider how placental supply capacity might be adapted and how the transfer of different solutes would be differentially affected. It will review the evidence, from human and mouse studies, that adaptation does indeed occur in response to mismatches between the ability of the placenta to supply nutrients and the fetal demand. Finally it will consider the implications of such adaptations for long-term programming of the physiology of the individual.

\section{Placental supply capacity - mechanisms of exchange across the placenta}

The barrier between maternal and fetal circulations in the human placenta consists of: (i) the syncytiotrophoblast, 
a multinucleated transporting epithelium which forms a complete layer throughout pregnancy; (ii) a layer of mononucleate cytotrophoblast cells which are predominantly progenitor cells for the syncytiotrophoblast and which seem to form a nearly complete layer in early pregnancy but by mid-pregnancy onwards form a discontinuous layer; (iii) connective tissue in the villous core and the fetal capillary endothelium, a normal continuous endothelium with lateral intercellular spaces which restrict the transfer of molecules with a molecular radius of about $1.5 \mathrm{~nm}$ upwards [4]. The syncytiotrophoblast is considered to be the main barrier and regulator of solute exchange across the barrier [1].

There are four main mechanisms of transfer across the barrier. Each of these will be utilized predominantly by different groups of solutes, dependent on their physicochemical characteristics. Any of the mechanisms may be altered to adapt the capacity of the placenta to supply nutrients.

\subsection{Bulk flow/solvent drag}

Differences in hydrostatic and osmotic pressures between the maternal and fetal microcirculations within the exchange barrier will drive water transfer by bulk flow, dragging with it dissolved solutes. These dissolved solutes will be filtered as they move through the components of the barrier. Water movement may be via paracellular channels [5] or across the plasma membranes. The latter may be enhanced by the presence of aquaporins, integral membrane proteins forming water 'pores' in the plasma membrane [6]. Adaptations in the flux by bulk flow/solvent drag may potentially occur by changes in the ratio of pre- and post-capillary resistances (an example is discussed below) or in the dimensions of the paracellular channel or membrane pores. Water flux will be predominantly altered by such changes.

\subsection{Diffusion}

Diffusion of any molecule occurs in both directions across the placental barrier. When there is a concentration gradient and/or, for charged species, an electrical gradient, one of these fluxes is greater than the other, so that there is a net flux in one direction. Net flux $\left(J_{\text {net }}\right)$ of solute across the placenta for an uncharged molecule may be described by an adaptation of Fick's Law of Diffusion [1]:

$J_{\text {net }}=(\mathrm{AD} / \mathrm{l})\left(C_{\mathrm{m}}-C_{\mathrm{f}}\right) \mathrm{mol} / \mathrm{unit}$ time

where $A$ is the surface area of the barrier available for exchange, $D$ is the diffusion coefficient in water of the molecule (smaller molecules will have larger $D$ ), $l$ is the thickness of the exchange barrier across which diffusion is occurring, $C_{\mathrm{m}}$ is the mean concentration of the molecule in maternal plasma and $C_{\mathrm{f}}$ is the mean concentration of solute in the fetal circulation.

Small, relatively hydrophobic molecules will diffuse rapidly across the plasma membranes of the barrier, so that their flux is dependent much more on the concentration gradient than on $A$ or $l$. Adaptations in blood flow on either side of the placenta will therefore predominantly affect flux of molecules such as $\mathrm{O}_{2}$ and $\mathrm{CO}_{2}$ [1]. By contrast, hydrophilic molecules will not diffuse across plasma membranes easily, their concentration gradients are maintained and flux will be determined predominantly by barrier surface area and thickness. The latter will include changes in the length and diameter of paracellular, water-filled channels across the syncytiotrophoblast (see [7] for discussion of evidence that such channels exist). Adaptations which affect these dimensions of the placental barrier will therefore predominantly affect flux of molecules such as ions, glucose and amino acids. Evidence that such adaptation does occur is discussed below.

\subsection{Transporter protein mediated processes}

Transporter proteins are integral membrane proteins which catalyse transfer of solutes across plasma membranes at faster rates than would occur by diffusion. Transporter proteins are a large and diverse group of molecules which are generally characterised by showing substrate specificity (i.e. one transporter or class of transporter will predominantly transfer one substrate or class of substrate e.g. amino acids), by having saturation kinetics (i.e. raising the concentration of a substrate solute will not infinitely increase the rate at which it is transferred on transporters) and by being competitively inhibitable (i.e. two structurally similar molecules will compete for transfer by a particular transporter protein). Transporter proteins are found abundantly in the placenta in the microvillous (maternal facing) and basal (fetal facing) plasma membranes of the syncytiotrophoblast. A detailed description of these may be found elsewhere [1]. Transporter proteins are highly regulatable, in terms of both expression and activity, and there is good evidence that such regulation does occur in the placenta. This may enable adaptations designed to meet specific requirements of the fetus for particular solutes; evidence of this is discussed in detail below.

\subsection{Endocytosis/exocytosis}

Endocytosis is the process by which molecules become entrapped in invaginations of the microvillous plasma membrane of the syncytiotrophoblast, which eventually pinch off and form vesicles within the cytosol. Such vesicles may move through the intracellular compartment and, if they avoid fusion with lysosomes, eventually fuse with the basal plasma membrane and undergo exocytosis, releasing their contents into the fetal milieu. Evidence suggests that immunoglobulin $\mathrm{G}(\operatorname{IgG})$ and other large proteins may cross the placenta by this mechanism [8]. Specificity and the ability to avoid lysosomal degradation during the endocytosis phase may be afforded by the presence of receptors for IgG in the microvillous plasma membrane invaginations and vesicles. As with transportermediated processes, this may enable adaptations that are designed to meet specific requirements of the fetus for particular solutes, although there is no evidence of such adaptation as yet.

\section{Evidence of adaptation of placental supply capacity to meet fetal demand in the human}

The first evidence from our laboratory of adaptation in human placental transporter activity in relation to fetal demand came from a study of System A amino acid transporter in microvillous plasma membranes of placentas from babies across the range of normal birthweights. We found that System A activity, per mg microvillous membrane protein, was highest in the smallest babies [9]. This was not what we expected as we had previously observed that System A activity, per mg microvillous membrane protein, was reduced in pregnancies affected by FGR [10]. This led us to postulate that in small normal babies placental System A activity can be upregulated to meet the demands of fetal growth and that one cause of FGR is a failure of such upregulation.

There is now considerable published data from studies of a variety of transporter activities in both the microvillous and basal plasma membranes of the syncytiotrophoblast from pregnancies affected by FGR. These data show that activities of some transporters (measured per mg microvillous membrane protein), like System A, are lower in membranes of placentas from FGR-affected pregnancies as compared to those from AGA babies [11]. Activity of at least one transporter, the $\mathrm{Ca}^{2+}$ ATPase, goes up [12]. Other transporters, such as the GLUT 1 glucose transporter [13], do not 
change at all (see also Table 1 of [7]). This range of changes suggests at least a degree of regulation with decreased activities contributing to the reduction of fetal growth and the increased $\mathrm{Ca}^{2+}$ ATPase activity perhaps being an adaptation in response to fetal demand in the face of decreased placental supply.

Interestingly, there is evidence that in normal pregnancy, the activity of some placental transporters increases over the course of gestation, e.g. System A [14], whilst that of others decreases, e.g. $\mathrm{Na}^{+} / \mathrm{H}^{+}$exchanger [14], perhaps reflecting adaptive responses to different fetal demands on the placenta as it develops and grows.

Is there any evidence that other determinants of placental supply capacity, other than transporter activity and expression, can adapt? One piece of data to suggest that there may be some regulation of blood flow in this way comes from a recently reported study of vascular endothelial growth factor (VEGF) production from the placenta, and its effects on its fetoplacental vasculature (Fig. 1). Brownbill et al. [15] found that the secretion of free VEGF into the fetoplacental circulation was lower in placentas from women suffering from pre-eclampsia than in those from women having normal pregnancies. However, as shown by the data in Fig. 1, while free VEGF was reduced with pre-eclampsia, its vasodilatory effects were greater. VEGF appears to be an important vasodilator of this circulation [16] and these data could reflect an increased sensitivity of the fetoplacental circulation to VEGF as an adaptive response to its reduced production, maintaining the paracrine regulation. However, caution needs to be observed in such an interpretation of these in vitro data as the increased sensitivity of pre-eclamptic placentas to VEGF only occurred at concentrations higher than those of free VEGF found in umbilical cord sera. As discussed by Brownbill et al. [15] a number of different factors will contribute to the overall contractility of the fetoplacental circulation in both health and disease.

Further support for the concept of adaptation in placental supply capacity to meet fetal demand comes from analysis of the relationship between placental and fetal weight. Heinonen et al. [17] reported that at any given birth weight, placental weight of small for gestational age (SGA) babies was significantly lower than that of appropriately grown for gestational age babies. This suggests that the placental transfer efficiency (grams of baby per gram placenta) was actually greater in the SGA group. Furthermore, Williams et al. [18] reported that the ratio of placental weight to birth weight does not accurately reflect fetal growth in normal pregnancy. This is consistent with the idea that several different determinants of placental supply capacity are adapted to meet fetal demand and that the mass of placental tissue per se is only one such determinant.

\section{Evidence for adaptation of placental supply capacity in the mouse}

Further evidence for the hypothesis that placental supply capacity adapts to meet fetal demand comes from mouse studies. Knockout of the placental specific transcript (P0) of the insulin-like growth factor 2 (Igf2) gene results in placental growth restriction at embryonic day 12 (e12) onwards (term is e20) but fetal growth restriction does not occur until around e18/e19 [19, 20]. This results in the fetal:placental weight ratio at e16 being 50\% higher in the P0 knockout conceptuses compared to wild type. When the maternofetal transfer of ${ }^{14} \mathrm{C}$-methylaminoisobutyric acid (MeAIB, a specific non-metabolizable substrate of System $A$ ) was measured in vivo, it was also found to be $50 \%$ increased, per g placenta, in the P0 placenta versus wild type. Interestingly, the same change was reflected in a $50 \%$ increase in the expression of the Slc38a4 isoform of the System A gene in the P0 knockout placentas [19, 20]. By contrast to these e16 data, at e19, when the P0 pups as well as placentas were growth restricted, all of these variables-fetal:placental weight ratio, ${ }^{14} \mathrm{C}$-MeAIB transfer and Slc38a4 mRNA-were similar in P0 knockout and wild type conceptuses. These data suggest that, as in the human study of Godfrey and colleagues [9], System A activity in the placenta can be upregulated to increase placental efficiency when the placental size per se is too low to meet the demands of the normally growing fetus. Such a change could also, at least partially, explain the human epidemiological data of Heinonen et al. [17] and Williams et al. [18]. However, the upregulation and increased efficiency does not appear to be sustainable in the mouse knockout, so that fetal growth restriction does finally ensue. As noted above, a failure to sustain placental adaptation in supply capacity could result in FGR in human pregnancy.

Coan et al. [21] prospectively investigated adaptation and increases in placental efficiency in normal mice. They compared the smallest and the largest placentas in normal mouse litters at e16 and e19. They found that there were increases in labyrinth zone (exchange region of the mouse placenta) volume fraction and increases in ${ }^{14} \mathrm{C}$-MeAIB transfer per $\mathrm{g}$ placenta in the smallest placentas. They also found changes in gene expression-upregulation of the Slc38a2 isoform of System A rather than of the Slc38a4 isoform-as found in the study described above. The authors speculated that these changes in labyrinth size and amino acid transport capacity enabled the small placentas to maintain normal fetal growth until term. Importantly, the morphological changes occurred at an earlier stage of gestation (e16) than did the transporter changes (e19), suggesting that the type of adaptation in
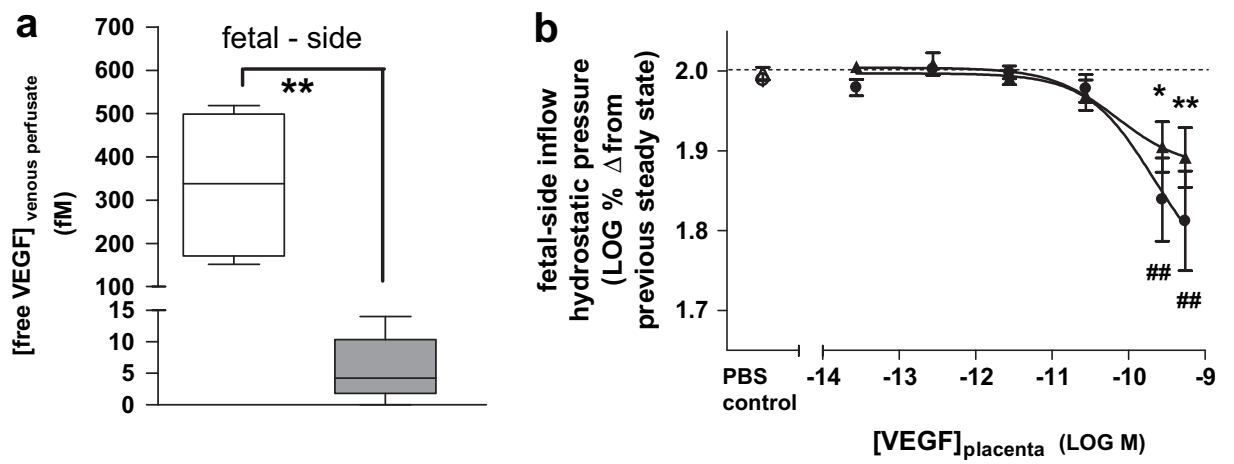

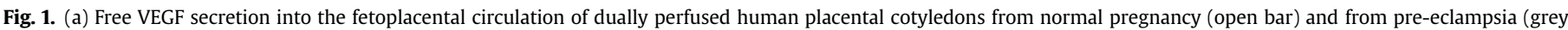

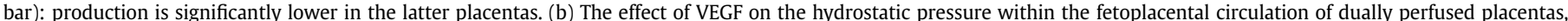

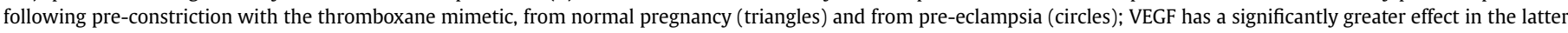
than the former. Data reproduced from [15], with permission, where further experimental details may be found. 
placental phenotype enabling increased efficiency can alter as pregnancy continues; we return to this concept and its implications below.

The change in $\mathrm{Ca}^{2+}$ ATPase activity in the basal plasma membrane of placentas from FGR pregnancies is the only increased transporter activity found to date, and is perhaps the most evocative of the concept of adaptation. As the P0 knockout mouse appeared to be, in many respects, a very good model of human FGR, we decided to investigate whether there was any alteration in calcium homeostasis in these conceptuses and whether there was any evidence of adaptation in placental calcium supply capacity. Our preliminary data ([22] and unpublished observations) show that plasma ionized calcium concentration is lower in the P0 fetuses than in their control wild type littermates at both e17 and e18. In fact the P0 fetuses apparently do not maintain the normal fetal greater than maternal ionized plasma concentration gradient. Furthermore, we found that fetal plasma calcium content (per g ash weight) was significantly lower in P0 fetuses compared to wild type at e17. However, there was no difference in calcium content at e19. These data show that PO conceptuses do indeed have an abnormality in calcium homeostasis reminiscent of that found in human FGR [23], especially at e17, but this is corrected, in terms of calcium accumulation if not circulating calcium concentrations, by e19.

We next investigated potential alterations in calcium transport across the placenta in relation to the altered homeostasis. Unidirectional maternofetal clearance of ${ }^{45} \mathrm{Ca}$ was found to be significantly higher in the P0 conceptuses than wild type at e 19 but not at e17. Investigation of the expression of the three components of calcium transport across the syncytiotrophoblast revealed that there was no difference between PO and wild type in the expression of the TrpV6 channel, through which calcium diffuses into the syncytiotrophoblast, or in expression of $\mathrm{Ca}^{2+}$ ATPase, which pumps calcium out of the syncytiotrophoblast into the fetal circulation. However, expression of calbindin- $\mathrm{D}_{9 \mathrm{k}}$ which binds calcium and shuttles it across the cell, was significantly lower in P0 placentas than wild type at e17 but was not different at e19 (Dilworth and Glazier, unpublished observations)

We interpret these data on calcium homeostasis in P0 knockout conceptuses as follows. It appears that they do not accumulate as much calcium in early to mid-pregnancy as do wild type conceptuses, as reflected in the calcium content of fetuses at e17 and the reduced fetal plasma calcium concentration. We are not sure of the reasons for this but it could reflect altered function of the choriovitelline placenta, which is formed earlier in pregnancy than the chorioallantoic placenta, and is known to be capable of transporting calcium [24]. We postulate that at about e17 this failure of calcium supply is signaled by the fetus to the placenta. This results in increased maternofetal calcium transfer perhaps mediated through the upregulation of calbindin- $D_{9 k}$, which is likely to be rate limiting for placental calcium transport in the rodent [25]. This adaptive upregulation of placental calcium transport results in fetal calcium content, per g ash weight, being normalized by e19.

There are two other points to emphasize concerning these observations. Firstly, in both human and mouse there is upregulation of a component of the placental calcium transport mechanism when fetal growth is restricted: in human this is $\mathrm{Ca}^{2+}$ ATPase activity, in mouse it is calbindin-D $\mathrm{Dk}_{\mathrm{k}}$. Secondly, it is interesting that the apparent adaptation of placental calcium supply to fetal demand for the cation occurs later in mouse gestation than does the upregulation in amino acid transport which, as described above, has occurred by e16 but which has returned close to normal by e19 $[19,20]$.

Putting all the evidence together we conclude that there is both observational and experimental evidence that placental nutrient supply capacity can be adapted in relation to fetal demand signals.
Further experimental evidence is now needed to examine this hypothesis further. The nature of the signals is another important question which now needs to be addressed, although some preliminary data perhaps suggest a role for glucocorticoids [26]. It should not be forgotten that the fetus itself can adapt to changes in its environment (e.g. increasing haemoglobin concentrations in the fetuses of sheep kept at high altitude [27]). How fetal adaptations and placental adaptations work together or independently to facilitate optimal fetal growth is a further interesting topic for future investigation.

\section{Implications for programming}

The previous sections describe evidence for adaptation in three components of placental supply capacity: blood flow, exchange barrier surface area and transporter activity and expression. In the future we need to try to build up data relating each of these to placental weight and size e.g. determining the total amino acid transporter activity in each placenta. From the consideration of mechanisms of placental exchange it is apparent that changes in blood flow will predominantly affect transfer of small hydrophobic solutes such as the respiratory gases. Changes in exchange surface area will have predominant effects on hydrophilic solutes such as glucose and amino acids. Transporter activity adaptations will have highly specific effects on transfer of particular solutes. The evidence from the mouse studies is that adaptations in particular components of supply capacity might occur at different stages of gestation. If this is so then the timing of birth will have a crucial effect on whether the fetus has been able to benefit from different components in an improvement in the matching of placental supply to fetal demand. Furthermore, we do not know whether each of these adaptations occur in concert or whether, in some pregnancies, e.g. only one component has had to be adapted to meet the needs of a particular fetus. As has also been commented upon by others [21], all of these effects will subtly and not so subtly alter the final composition of a baby (fat versus lean, concentrations of metabolites such as amino acids, glucose, calcium in body fluid compartments, etc.). We postulate that such changes will alter homeostatic set points with life-long effects on how the individual reacts to changes in his or her environment. A considerable amount of work is needed to test whether this is indeed one pathway of in utero programming.

\section{Conflict of interest}

The authors do not have any potential or actual personal, political, or financial interest in the material, information, or techniques described in this paper.

\section{Acknowledgements}

This work was supported by The Wellcome Trust, an Action Research Endowment Fund and Tommy's Charity.

\section{References}

[1] Atkinson D, Boyd R, Sibley C. Placental transfer. In: Neill JD, editor. Knobil and Neill's physiology of reproduction. 3rd ed. Elsevier; 2006. p. 2787-846.

[2] Catalano PM, Ehrenberg HM. The short- and long-term implications of maternal obesity on the mother and her offspring. BJOG 2006;113:1126-33.

[3] Godfrey KM, Barker DJ. Fetal programming and adult health. Public Health Nutr $2001 ; 4: 611-24$

[4] Firth JA, Leach L. Not trophoblast alone: a review of the contribution of the fetal microvasculature to transplacental exchange. Placenta 1996;17:89-96.

[5] Brownbill P, Mahendran D, Owen D, Swanson P, Thornburg KL, Nelson DM, et al. Denudations as paracellular routes for alphafetoprotein and creatinine across the human syncytiotrophoblast. Am J Physiol Regul Integr Comp Physiol 2000;278:R677-83. 
[6] Liu H, Koukoulas I, Ross MC, Wang S, Wintour EM. Quantitative comparison of placental expression of three aquaporin genes. Placenta 2004;25:475-8.

[7] Sibley CP. Understanding placental nutrient transfer-why bother? New biomarkers of fetal growth. J Physiol 2009;587:3431-40.

[8] Lin CT. Immunoelectron microscopy localization of immunoglobulin $G$ in human placenta. J Histochem Cytochem 1980;28:339-46.

[9] Godfrey KM, Matthews N, Glazier J, Jackson A, Wilman C, Sibley CP. Neutral amino acid uptake by the microvillous plasma membrane of the human placenta is inversely related to fetal size at birth in normal pregnancy. J Clin Endocrinol Metab 1998;83:3320-6.

[10] Mahendran D, Donnai P, Glazier JD, D’Souza SW, Boyd RD, Sibley CP. Amino acid (system $A$ ) transporter activity in microvillous membrane vesicles from the placentas of appropriate and small for gestational age babies. Pediatr Res 1993;34:661-5.

[11] Jansson T, Scholtbach V, Powell TL. Placental transport of leucine and lysine is reduced in intrauterine growth restriction. Pediatr Res 1998;44:532-7.

[12] Strid H, Bucht E, Jansson T, Wennergren M, Powell TL. ATP dependent $\mathrm{Ca}^{2+}$ transport across basal membrane of human syncytiotrophoblast in pregnancies complicated by intrauterine growth restriction or diabetes. Placenta 2003;24:445-52.

[13] Jansson T, Wennergren M, Illsley NP. Glucose transporter protein expression in human placenta throughout gestation and in intrauterine growth retardation. J Clin Endocrinol Metab 1993;77:1554-62.

[14] Mahendran D, Byrne S, Donnai P, D’Souza SW, Glazier JD, Jones CJ, et al. $\mathrm{Na}^{+}$ transport, $\mathrm{H}^{+}$concentration gradient dissipation, and system $\mathrm{A}$ amino acid transporter activity in purified microvillous plasma membrane isolated from first-trimester human placenta: comparison with the term microvillous membrane. Am J Obstet Gynecol 1994;171:1534-40.

[15] Brownbill P, Mills TA, Soydemir DF, Sibley CP. Vasoactivity to and endogenous release of vascular endothelial growth factor in the in vitro perfused human placental lobule from pregnancies complicated by preeclampsia. Placenta 2008;29:950-5.

[16] Brownbill P, McKeeman GC, Brockelsby JC, Crocker IP, Sibley CP. Vasoactive and permeability effects of vascular endothelial growth factor-165 in the term in vitro dually perfused human placental lobule. Endocrinology 2007;148:4734-44.
[17] Heinonen S, Taipale P, Saarikoski S. Weights of placentae from small-forgestational age infants revisited. Placenta 2001;22:399-404.

[18] Williams LA, Evans SF, Newnham JP. Prospective cohort study of factors influencing the relative weights of the placenta and the newborn infant. BM] (Clin Res Ed) 1997;314:1864-8.

[19] Constancia M, Angiolini E, Sandovici I, Smith P, Smith R, Kelsey G, et al Adaptation of nutrient supply to fetal demand in the mouse involves interaction between the Igf2 gene and placental transporter systems. Proc Nat Acad Sci USA 2005;102:19219-24.

[20] Constancia M, Hemberger M, Hughes J, Dean W, Ferguson-Smith A, Fundele R, et al. Placental-specific IGF-II is a major modulator of placental and fetal growth. Nature 2002;417:945-8.

[21] Coan PM, Angiolini E, Sandovici I, Burton GJ, Constancia M, Fowden AL. Adaptations in placental nutrient transfer capacity to meet fetal growth demands depend on placental size in mice. J Physiol 2008;586:4567-76.

[22] Dilworth MR, Cowley E, Husain S, Ward B, Sibley C, Constancia M, et al. Placental specific Igf2 (P0) knockout mice demonstrate hypocalcaemia and gestational dependent changes in calcium content. Reprod Sci 2009; 16:343A.

[23] Kramer MS, Olivier M, McLean FH, Willis DM, Usher RH. Impact of intrauterine growth retardation and body proportionality on fetal and neonatal outcome. Pediatrics 1990;86:707-13.

[24] Bruns ME, Kleeman E, Mills SE, Bruns DE, Herr JC. Immunochemical localization of vitamin D-dependent calcium-binding protein in mouse placenta and yolk sac. Anatomical Rec 1985;213:514-7. pp. 32-35.

[25] Glazier JD, Atkinson DE, Thornburg KL, Sharpe PT, Edwards D, Boyd RD, et al. Gestational changes in $\mathrm{Ca} 2+$ transport across rat placenta and mRNA for calbindin9K and $\mathrm{Ca}(2+)$-ATPase. Am J Physiol 1992;263:R930-5.

[26] Vaughan O, Sferruzzi-Perri A, Coan P, Burton G, Fowden A. Effects of maternal dexamethasone treatment on amino acid clearance and diffusional exchange capacity of the mouse placenta depend on gestational age. Placenta 2009; 30:A.11.

[27] Kitanaka T, Alonso JG, Gilbert RD, Siu BL, Clemons GK, Longo LD. Fetal responses to long-term hypoxemia in sheep. Am J Physiol 1989;256: R1348-54. 